\title{
Minimum information about tolerogenic antigen-presenting cells (MITAP): a first step towards reproducibility and standardisation of cellular therapies
}

Phillip Lord Corresp.. 1 , Rachel Spiering 2 , Juan C Aguillon ${ }^{3}$, Amy E Anderson ${ }^{2}$, Silke Appel ${ }^{4}$, Daniel BenitezRibas $^{5}$, Anja ten Brinke ${ }^{6}$, Femke Broere ${ }^{7}$, Nathalie Cools ${ }^{8}$, Maria Cristina Cuturi ${ }^{9}$, Julie Diboll ${ }^{2}$, Edward K Geissler ${ }^{10}$, Nick Giannoukakis ${ }^{11}$, Silvia Gregori ${ }^{12}$ ， S. Marieke van Ham ${ }^{6}$, Staci Lattimer ${ }^{1}$, Lindsay Marshall $^{1}$, Rachel A Harry ${ }^{2}$, James A Hutchinson ${ }^{10}$ ， John D Isaacs ${ }^{2}$, Irma Joosten ${ }^{13}$, Cees van Kooten ${ }^{14}$, Ascension Lopez Diaz de Cerio ${ }^{15}$, Tatjana Nikolic ${ }^{16}$, Haluk Barbaros Oral ${ }^{17}$, Ljiljana Sofronic-Milosavljevic ${ }^{18}$, Thomas Ritter ${ }^{19}$ ， Paloma Riquelme ${ }^{10}$, Angus W Thomson ${ }^{20}$, Massimo Trucco ${ }^{11}$, Marta Vives-Pi ${ }^{21,22}$, Eva Martinez-Caceres ${ }^{21}$, Catharien MU Hilkens ${ }^{\text {Corresp. } 2}$

1 School of Computing Science, Newcastle University, Newcastle upon Tyne, United Kingdom

2 Institute of Cellular Medicine, Newcastle University, Newcastle upon Tyne, United Kingdom

3 Instituto de Ciencias Biomédicas (ICBM), Universidad de Chile, Santiago, Chile

4 Broegelmann Research Laboratory, Department of Clinical Science, University of Bergen, Bergen, Norway

5 Department of Immunology, Hospital Clínic i Provincial and Centro de Investigación Biomédica en Red de Enfermedades Hepáticas y Digestivas (CIBERehd), Barcelona, Spain

6 Department of Immunopathology, Sanquin Research, Amsterdam, The Netherlands

7 Faculty of Veterinary Medicine, Department of Infectious Diseases and Immunology, Utrecht University, Utrecht, The Netherlands

8 Laboratory of Experimental Hematology, Vaccine \& Infectious Disease Institute (VAXINFECTIO), Faculty of Medicine and Health Sciences, University of Antwerp, Wilrijk, Belgium

9 Center for Research in Transplantation and Immunology, ITUN, Inserm UMRS 1064, Nantes, France

10 Department of Surgery, University Hospital Regensburg, Regensburg, Germany

11 Institute of Cellular Therapeutics, Pittsburgh, United States of America

12 San Raffaele Telethon Institute for Gene Therapy (SR-Tiget), Division of Regenerative Medicine, Stem Cells and Gene Therapy,, IRCCS San Raffaele Scientific Institute (DREaM-GENE), Milan, Italy

13 Department of Laboratory Medicine, Radboud University Nijmegen Medical Center, Nijmegen, The Netherlands

14 Department of Nephrology, Leiden University Medical Centre, Leiden, The Netherlands

15 Area of Cell Therapy, University Clinic of Navarra, Pamplona, Spain

16 Department of Immunohematology and Blood Transfusion, Leiden University Medical Centre, Leiden, The Netherlands

17 Department of Immunology, Faculty of Medicine, Uludag University, Bursa, Turkey

18 Institute for the Application of Nuclear Energy INEP, University of Belgrade, Belgrade, Serbia

19 Regenerative Medicine Institute (REMEDI), School of Medicine, College of Medicine, Nursing and Health Sciences, National University of Ireland, Galway, Ireland

20 Thomas E. Starzl Transplantation Institute, University of Pittsburgh School of Medicine, Pittsburgh, United States of America

21 Immunology Division, Germans Trias i Pujol University Hospital and Health Sciences Research Institute, Badalona, Spain

22 CIBER of Diabetes and Associated Metabolic Diseases (CIBERDEM), Instituto de Salud Carlos III (ISCIII), Madrid, Spain

Corresponding Authors: Phillip Lord, Catharien MU Hilkens

Email address: phillip.lord@newcastle.ac.uk, catharien.hilkens@ncl.ac.uk

Cellular therapies with tolerogenic antigen-presenting cells (tolAPC) show great promise for the treatment of autoimmune diseases and for the prevention of destructive immune responses after transplantation. The methodologies for generating tolAPC vary greatly between different laboratories, making it difficult to compare data from different studies; thus constituting a major hurdle for the development of standardised tolAPC therapeutic 
products. Here we describe an initiative by members of the tolAPC field to generate a minimum information model for tolAPC (MITAP), providing a reporting framework that will make differences and similarities between tolAPC products transparent. In this way MITAP constitutes a first but important step towards the production of standardised and reproducible tolAPC for clinical application. 
1 Minimum information about tolerogenic antigen-presenting cells

2 (MITAP): a first step towards reproducibility and standardisation

3 of cellular therapies

4

Phillip Lord ${ }^{1 *}$, Rachel Spiering ${ }^{2}$, Juan C. Aguillón ${ }^{3}$, Amy E. Anderson ${ }^{2}$, Silke Appel ${ }^{4}$, Daniel Benitez-Ribas $^{5}$, Anja ten Brinke ${ }^{6}$, Femke Broere ${ }^{7}$, Nathalie Cools ${ }^{8}$, Maria Cristina Cuturi ${ }^{9}$, Julie Diboll $^{2}$, Edward K. Geissler ${ }^{10}$, Nick Giannoukakis ${ }^{11}$, Silvia Gregori ${ }^{12}$, S. Marieke van Ham ${ }^{6}$, Staci Lattimer $^{1}$, Lindsay Marshall ${ }^{1}$, Rachel A. Harry ${ }^{2}$, James A. Hutchinson ${ }^{10}$, John D. Isaacs ${ }^{2}$, Irma Joosten $^{13}$, Cees van Kooten ${ }^{14}$, Ascensión López Díaz de Cerio ${ }^{15}$, Tatjana Nikolic ${ }^{16}$, Haluk Barbaros Oral $^{17}$, Ljiljana Sofronic-Milosavljevic ${ }^{18}$, Thomas Ritter ${ }^{19}$, Paloma Riquelme ${ }^{10}$, Angus W. Thomson ${ }^{20}$, Massimo Trucco ${ }^{11}$, Marta Vives-Pi ${ }^{21,22}$, Eva Martinez-Caceres ${ }^{21}$, Catharien M.U. Hilkens ${ }^{2 *}$

${ }^{1}$ School of Computing Science and ${ }^{2}$ Institute of Cellular Medicine, Newcastle University, NE2 4HH, Newcastle upon Tyne, U.K.

${ }^{3}$ Immunology Program, Instituto de Ciencias Biomédicas (ICBM), Facultad de Medicina, Universidad de Chile, Santiago, Chile

${ }^{4}$ Broegelmann Research Laboratory, Department of Clinical Science, University of Bergen, Bergen, Norway

${ }^{5}$ Dept. of Immunology, Hospital Clínic i Provincial and Centro de Investigación Biomédica en Red de Enfermedades Hepáticas y Digestivas (CIBERehd), Barcelona, Spain

${ }^{6}$ Sanquin Research, Dept Immunopathology, Amsterdam, The Netherlands, and Landsteiner Laboratory, Academic Medical Centre, University of Amsterdam, Amsterdam, The Netherlands ${ }^{7}$ Faculty of Veterinary Medicine, Department of Infectious Diseases and Immunology, Utrecht University, 3584 CL, Utrecht, The Netherlands

${ }^{8}$ Laboratory of Experimental Hematology, Vaccine \& Infectious Disease Institute (VAXINFECTIO), Faculty of Medicine and Health Sciences, University of Antwerp, 2610

Wilrijk, Belgium

${ }^{9}$ ITUN, Inserm UMRS 1064, Center for Research in Transplantation and Immunology, Nantes, France

${ }^{10}$ Department of Surgery, University Hospital Regensburg, Franz-Josef-Strauß-Allee-11, 93053 Regensburg, Germany

${ }^{12}$ Institute of Cellular Therapeutics, Allegheny Health Network, Pittsburgh, PA, 15215, USA

${ }^{12}$ San Raffaele Telethon Institute for Gene Therapy (SR-Tiget), Division of Regenerative Medicine, Stem Cells and Gene Therapy, IRCCS San Raffaele Scientific Institute (DREaMGENE), Via Olgettina 58, Milan, Italy

${ }^{13}$ Department of Laboratory Medicine, Radboud University Nijmegen Medical Center, PO Box 9101, 6500HB

Nijmegen, The Netherlands

${ }^{14}$ Dept of Nephrology, LUMC, Leiden, The Netherlands 
$41{ }^{15}$ Area of Cell Therapy, University Clinic of Navarra, Pamplona, Spain

$42{ }^{16}$ Department of Immunohematology and Blood Transfusion, Leiden University Medical Center,

43 2333ZA Leiden, The Netherlands

$44{ }^{17}$ Department of Immunology, Faculty of Medicine, Uludag University, Nilufer-16059, Bursa,

45 Turkey

$46{ }^{18}$ Institute for the Application of Nuclear Energy INEP, University of Belgrade, Banatska 31b,

47 11080, Belgrade, Serbia

$48{ }^{19}$ Regenerative Medicine Institute (REMEDI), School of Medicine, College of Medicine, Nursing

49 and Health Sciences, National University of Ireland, Galway, Ireland

$50{ }^{20}$ Thomas E. Starzl Transplantation Institute, University of Pittsburgh School of Medicine, 200

51 Lothrop Street, W1544 Biomedical Science Tower, Pittsburgh PA 15261

$52{ }^{21}$ Immunology Division, Germans Trias i Pujol University Hospital and Health Sciences

53 Research Institute. Campus Can Ruti. Autonomous University of Barcelona, 08916,

54 Badalona, Spain

$55{ }^{22}$ CIBER of Diabetes and Associated Metabolic Diseases (CIBERDEM), Instituto de Salud

56 Carlos III (ISCIII), 28029, Madrid, Spain

57

58 Corresponding authors:

59 Phillip Lord: phillip.lord@ncl.ac.uk

60 Catharien Hilkens: catharien.hilkens@ncl.ac.uk

61

$62 *$ These authors contributed equally

63

64 
65 Abstract

66 Cellular therapies with tolerogenic antigen-presenting cells (tolAPC) show great promise for the

67 treatment of autoimmune diseases and for the prevention of destructive immune responses after

68 transplantation. The methodologies for generating tolAPC vary greatly between different

69 laboratories, making it difficult to compare data from different studies; thus constituting a major

70 hurdle for the development of standardised tolAPC therapeutic products. Here we describe an

71 initiative by members of the tolAPC field to generate a minimum information model for tolAPC

72 (MITAP), providing a reporting framework that will make differences and similarities between

73 tolAPC products transparent. In this way MITAP constitutes a first but important step towards

74 the production of standardised and reproducible tolAPC for clinical application.

Introduction

77 Immunotherapy with whole living cells shows great promise for the treatment of a wide variety

78 of complex diseases. A recent innovation in this field is the use of myeloid antigen-presenting

79 cells (APC) with immunoregulatory function to treat autoimmune diseases, or to prevent

80 destructive immune reactions after organ - or haematopoietic stem cell-transplantation 1,2,3,4,5,6,7,

$81{ }^{8}$. Examples of these immunoregulatory APC include tolerogenic dendritic cells (tolDC) and

82 regulatory macrophages (Mreg). The main advantage of these tolerogenic/regulatory APC

83 (hereafter referred to as tolAPC) therapies over conventional immunosuppressive drugs is that

84 these cells are uniquely equipped to specifically target harmful T-cell responses to auto- or allo-

85 antigens. Thus, tolAPC act in an antigen-specific manner and do not cause general

86 immunosuppression after exposure to antigen or in the allogeneic transplant setting - they are,

87 therefore, less likely to affect protective immune responses to invading pathogens. More

88 remarkably, as tolAPC induce or restore immune tolerance, and immune tolerance is a self-

89 reinforcing state, the beneficial effects of tolAPC treatment are expected to continue beyond the

90 lifespan of these therapeutic cells. Thus, tolAPC therapy holds the promise of a potentially

91 curative treatment with low toxicity.

93 A variety of therapeutic tolAPC products have been developed over the last decade, and a

94 number of these have recently been tested, or are undergoing testing in the clinic. Phase I trials

95 with autologous tolAPC have been completed for type I diabetes ${ }^{9}$, rheumatoid arthritis ${ }^{10}$ and 
96 Crohn's disease ${ }^{11}$, and further phase I trials for multiple sclerosis, allergic asthma and kidney

97 transplantation are in progress ${ }^{8}$. So far the results are highly encouraging from a safety standpoint, and further phase II clinical trials to test the efficacy of tolAPC therapies are under development, with the first tolDC phase II trial in type I diabetes recruiting imminently.

An outstanding and important issue within the tolAPC field is the variation in the methods used for cell isolation and culture. Current protocols to produce tolDC or Mreg involve the isolation of peripheral blood $\mathrm{CD} 14^{+}$monocytes followed by cell culture under specific conditions to generate the desired type of tolAPC. For example, monocytes can be differentiated into DC by culturing them in the presence of the cytokines IL-4 and GM-CSF, and stable tolerogenic function can be induced in these DC by treatment with immunosuppressive compounds (e.g. dexamethasone, IL10) or genetic modification to silence immunostimulatory molecules. In addition to compound variety, the concentration used may vary, as well as the timing of administration to the tolAPC cultures. These almost endless variations in the methodology make comparison of different tolAPC products difficult, therefore bringing real uncertainty when comparing safety and efficacy results from different clinical trials, testing different tolAPC products in different

112 diseases/conditions.

\section{Minimum Information Models (MIMs)}

115 Minimum Information Models (MIMs) are now being recognised as an important tool for the scientific community to use and interpret reported data, enabling comparison between data from different studies and facilitating experimental reproducibility. While it is simple for different laboratories to share and exchange their data, for this sharing to be meaningful, allowing reuse and repurposing, it must share some commonality of reporting, methodology or interpretation. This process has recently raised much interest, for example with the 5-star open data initiative (http://5stardata.info). A MIM is the first step in this process, providing a mechanism for the broadest level of commonality, ensuring that different laboratories report at least the key facts about their analysis in a clear and consistent manner, by providing a document and checklist

124 describing the necessary information. They are simple and easy-to-use, so are supportive rather a microarray experiment) ${ }^{12}$ and the more recent MIATA (minimal information about T-cell 
127 assays) ${ }^{13,14}$, the latter now actively being endorsed by seven international scientific journals. As

128 well as allowing sharing of data between individual laboratories, when widely used, they also 129 provide an excellent basis for building large integrated resources of data that can be searched 130 efficiently and comprehensively.

\section{Setting up MITAP: Community Building and Initial Analysis}

134 Our goal was to provide a reporting framework that will make differences and similarities of tolAPC transparent. We therefore set out to create minimum reporting guidelines for the production process of tolAPC used in pre-clinical and/or clinical studies. We call this MITAP (Minimum Information about Tolerogenic Antigen-Presenting cells).

A critical part of building the MITAP document was community building; to enable data sharing and commonality, the document must be broadly reflective of the opinions and current practices of many laboratories within the discipline. For this, a community is needed to provide information, feedback and tests subjects. Helpfully, we recently established a consortium of investigators working in the field of tolerogenic cellular therapies, with the aim of jointly addressing issues related to the translation and clinical application of these new treatments. This consortium is called AFACTT (action to focus and accelerate cell-based tolerance-inducing therapies - http://www.afactt.eu). It convenes every 6 months at interactive workshops. This consortium provided an ideal vehicle for the process of developing MITAP, which we achieved through a series of "exercises" that introduced AFACTT members to the general notion of MIMs, as well as providing some initial data, which we used to scope the MITAP document. We used three main forms of exercise both of which aimed at gathering "terms" -- basic vocabulary in use within the community. The first is called the "sticky-note" exercise which we performed at several AFACTT meetings: for this, each participant writes a term on a sticky-note; these are then collated and clustered on a wall by the whole group, identifying synonyms and related terms. Secondly, we gathered bibliographic databases from all willing AFACTT participants; abstracts were gathered from PubMed, and analysed computationally to find words that were over-represented in these abstracts compared to the bulk of PubMed. Finally, we built some 
157 bespoke software to crowd-source community feedback on these terms using a simple web 158 interface.

The conception of MITAP took place in April 2014, at the first AFACTT meeting; the overall process of creating the MITAP document took 18 months (Figure 1), which reflects the nature of the process i.e. the active involvement of a diverse group of investigators and the absolute need for incorporating feedback and suggestions from this group.

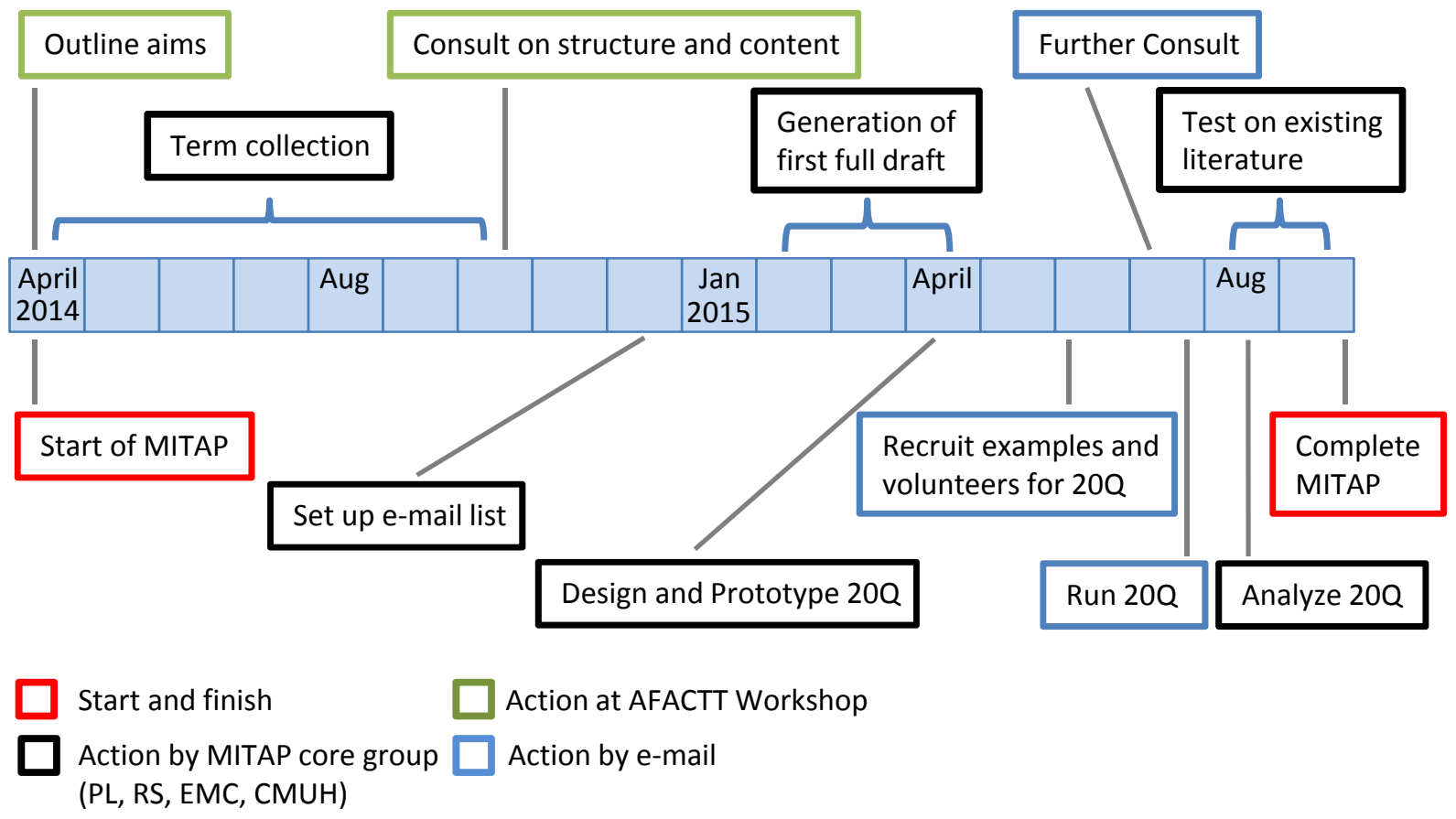

Figure 1. MITAP time line: from start to finish. 20Q: the "20 questions" experiment.

\section{Overview of the MITAP document}

167 The MITAP document is divided into 4 sections that encapsulate the most important parts of the tolAPC production process in a chronological manner. The sections describe the cells at the start of the production process, during the differentiation/induction of tolerogenicity process, and at the end of the production process, prior to injection into the recipient or use in experimental

171 assays. A final section is dedicated to aspects that are important for understanding the application 172 and purpose of tolAPC. The sections are described in more detail below; for all the sections and 173 subsections the document states clearly whether the investigator must (required), should (if 174 available) or may (optional) provide the relevant information. It also advises on the use of 
175 existing taxonomies and databases to provide the information in a uniform manner, and it

176

177

178

179

180

181

182

183

184

185

186

187

188

189

190

191

192

193

194

195

196

197

198

199

200

201

202

203

204

205

suggests the use of other MIMs where appropriate. The full MITAP document can be found on the AFACTT website (http://www.afactt.eu; see also Section 'Sustainability' below).

Section 1. Cells Before. This section describes the characteristics and state of the cells before they undergo any manipulation to become a tolAPC. There are five subparts to this section. First, it asks for essential information about the donor, including the species (surprisingly not always mentioned in research papers), strain if working with experimental animals, and any characteristics of the organism that are deemed to have a possible impact on the tolAPC production process (e.g. age or sex of the organism, or whether they are taking medication). Second, the tissue, organ or fluid from which the cells are used need to be described. In most published tolAPC studies the starting material is bone marrow or peripheral blood, but it is conceivable that for some applications investigators may want to isolate cells from specific organs (e.g. spleen). For human studies, peripheral blood products are the most often used source; these include freshly drawn blood by venepuncture, leukapheresis products and buffy coats (the latter can be purchased from Bloodbanks). Third, if investigators extract a subpopulation of cells from the initial cell source then information about the extraction method and the equipment should be provided. Fourth, the phenotype of the extracted cells should be described (e.g. morphology, expression of cell markers); in addition, the proportion of the cells that display a certain characteristic needs to be reported, to provide important information on the uniformity ('purity') of the cell population. In the last subpart of this section, details on the absolute cell number and cell viability should be provided.

Section 2. Differentiation and Induction of Tolerogenicity. This section describes the protocol that has been used to differentiate and/or induce tolerogenicity in the cells described in section 1 . It comprises 5 parts. The first part describes the pre-culture conditions the cells are being kept in before the start of the cell culture process to generate tolAPC. This may include freezing and thawing of the cells. Second, the culture conditions of the cells should be provided, including the starting cell number, cell concentration, culture medium, culture container, and the culture environment (e.g. temperature, $\mathrm{CO}_{2}$ levels). The third part deals with the differentiation/induction of tolerogenic function in the cells. It should be noted here that 
206

207

208

209

210

211

212

213

214

215

216

217

218

219

220

221

222

223

224

225

226

227

228

229

230

231

232

233

234

235

236

differentiation and induction of tolerogenicity are not necessarily interchangeable; they can be seen as distinct processes. For example, tolAPC have been generated by differentiation of CD14 ${ }^{+}$ monocytes into immature or semi-mature DC; thus, without any active induction of pro-

tolerogenic properties in the cells. In contrast, other protocols rely on the use of specific agents to induce stable tolerogenic function in DC during differentiation from precursor cells, and thus will use agents for both the differentiation and conferral of tolerogenicity of/in cells. In the fourth part investigators are asked to describe the antigen (if any) they use to load the tolAPC; this part is more likely to apply to investigators working in the field of autoimmunity, where tolAPC need to be targeted to certain autoantigen(s), and less so to the field of transplantation, which often (but not exclusively) uses donor-derived tolAPC already expressing the relevant allogeneic MHC/peptide complexes. Use of autologous APCs pulsed with donor antigen, e.g. in the form of donor cell lysate or exosomes (that accommodates the ${ }^{1}$ polymorphic MHC) is also possible. The final part of this section is about storage of the cells. If tolAPC are administered 'freshly', the conditions under which the cells are being kept in between harvesting and injection into the recipient or use in experimental assays need to be described. On the other hand, if tolAPC are being frozen, the process of freezing and thawing needs to be described.

3) Cells After. This section describes the characteristics and state of the cells after the differentiation/induction of tolerogenicity process has taken place. Two of the parts (part 1 and 3 ) cover the same type of information as in section 1, i.e. details on the cell phenotype, number and viability. The second part of this section provides details on any characteristics of the cells that have been measured by means of a functional in vitro assay. This can include details on their migratory capacity, or their ability to induce T regulatory cells.

4) About the protocol. The final section of MITAP describes the general features of the protocol as a whole. The first part provides information about any external regulatory authorities that have approved the study, and whether the cells were produced under GLP or GMP guidelines. Second, the purpose of the produced tolAPC should be described, e.g. prevention of transplant rejection or restoring immune tolerance in an autoimmune disease. The relationship between the cells and the target organism should be described i.e. allogeneic, autologous, xenogeneic or syngeneic. Finally, the name and contact details of the corresponding author must be provided. 
238 The MITAP document is accompanied by a handy checklist to assist investigators in ensuring that all the relevant detail is provided before submitting their manuscripts for publication.

\section{Testing MITAP: The “20 Questions” Experiment}

The MITAP document was developed collaboratively by scientists in the AFACTT consortium and should, therefore, be usable by this community. However, we wished to test directly whether the document as it stood was comprehensible by individuals from the discipline, and that data could be entered in a consistent manner. We therefore performed a "question-and-answer" experiment, a variation on the spoken parlour game "20 questions", popular in the $19^{\text {th }}$ and $20^{\text {th }}$ centuries. We collected a list of terms - that is short descriptive phrases that might be found in a description of a procedure to generate tolAPC, each of which would fit into one of the categories defined by the MITAP document. We then asked a number of scientists from the discipline, our test subjects, to place these terms into the relevant category from MITAP, which we call the test task. The basic premise of the experiment is simple: if the document is clear and comprehensible, then different test subjects should independently perform this task consistently.

The experiment was designed following a number of preconditions. First, the test task could not be too onerous for the test subjects, so that we could maximise completion rates; in particular, we felt that the task should take less than 30 minutes (including reading documentation). Our initial trials suggested that a subject could place two phrases for each category in MITAP in this time. Second, our terms should be representative of the discipline, rather than a single group, as far as was possible. Therefore, we asked members of the AFACTT group to contribute these terms, limited to two for each section in MITAP; for the experiment, we selected a subset of these terms randomly, with minor alterations for length. Third, our test subjects should also be representative of the discipline. Therefore, we asked those who contributed terms to also nominate a test subject. Finally, the test subjects were people who had not directly contributed to MITAP, nor seen the document, nor were involved in contributing terms.

For the test itself, test subjects were presented with a set of terms and for each asked to assess into which category it should be placed. Test subjects were given no guidance on how to 
268 categorise terms except for the MITAP document. In fact, they were given a slightly reduced

269 MITAP document; the non-normative (i.e. explanatory and not a strict part of the standard)

270 examples were removed for the purpose of this experiment. Subjects were also asked for some

271 basic metadata (level of experience, discipline and so forth). The test was conducted using an

272 online survey system (Google forms). Strictly, this means that we could not enforce lack of

273 collaboration; we considered this compromise to be appropriate, as it increased the number of

274 test subjects and their spread across the discipline.

275

“20 Questions" Results

277 The question and answer experiment was conducted over a two-week period in July 2015. We

278 invited 24 test subjects to take part and received results from 23, representing a 96\% completion

279 rate. Each subject categorized 44 terms from which example terms are "xenogeneic", "Rattus

280 norvegicus" or "immunomagnetic separation".

281

282 For the test task, subjects could ascribe each term to any number of MITAP categories. A

283 number of categories in MITAP are repetitive (cell numbers before and after the

284 differentiation/induction of tolerogenicity process, for example). These categories were

285 normalized for most of the results presented below. Answers from test subjects were compared

286 against our "Gold Standard" - the answers defined by primary authors of the MITAP document.

287 As subjects could give multiple answers, we assessed answers as either being exactly the same as

288 the gold standard, or containing at least the gold standard (Figure 2). 


\section{Inter-annotator agreement}

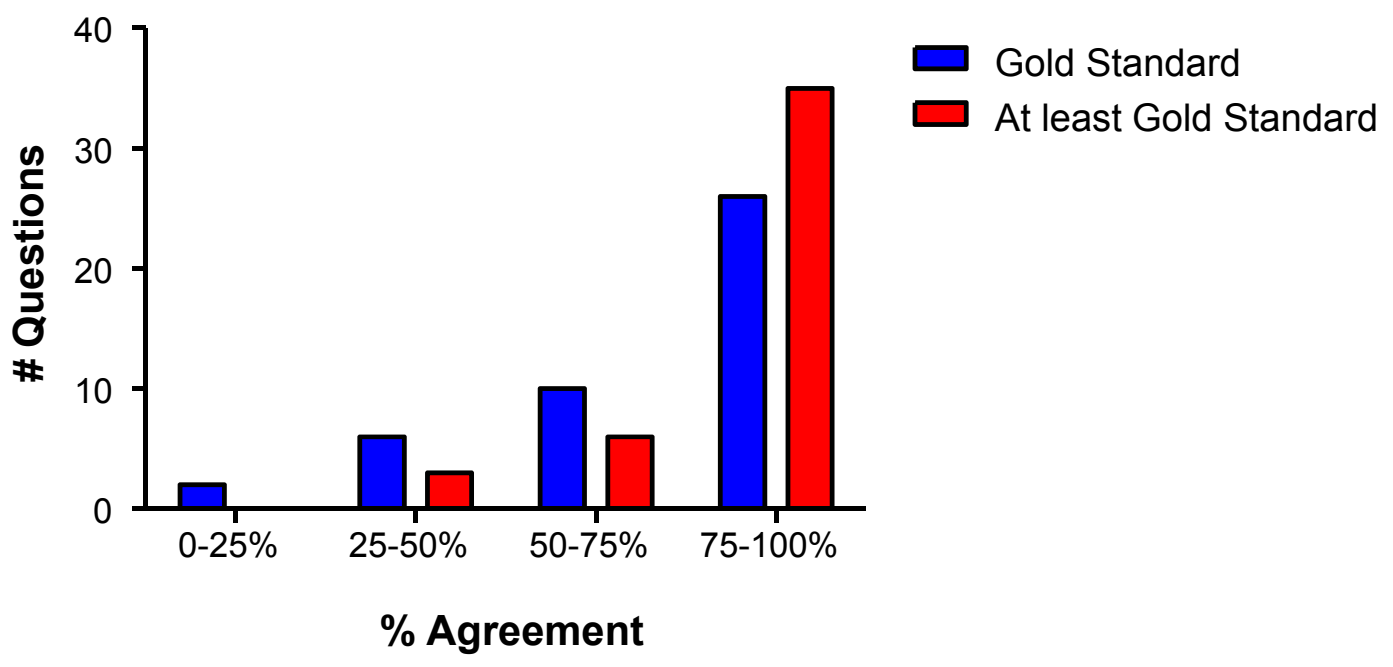

Figure 2: Summary of the results of the "20 Questions" Experiment. The answers of the test subjects were divided over four quadrants: 0-25\%, 25-50\%, 50-75\% and 75-100\%, where e.g. 0$25 \%$ means that the number of questions that were only answered correctly by $0-25 \%$ of the test subjects and $75-100 \%$ meaning that these questions were answered correctly by $75-100 \%$ of the participants. A total of 44 questions were presented to test subjects. In blue, we show only answers with exactly the Gold Standard as correct. In red, we show answers with at least the Gold Standard plus any number of other answers as correct.

Broadly, the results showed a very high-level of test subject agreement, averaging at around 73\% for Gold Standard only and $84 \%$ for at least Gold Standard. Terms that were put in the right category by all test subjects included 'immunomagnetic separation' (Section 1. Cell separation process) and 'Spanish regulatory agency (AEMPS)' (Section 4. Regulatory Authority). Terms that test subjects were struggling with (0-50\% agreement) included "cytomegalovirus (CMV)" for which the Gold Standard was antigen (see Section 2. Differentiation and Induction of Tolerogenicity), but that could also correctly be put under 'characteristics of the organism'

311 (Section 1). Another example of a term often placed under the wrong subheading was: "mice 312 were obtained from the Jackson Laboratory", which was often placed under 'species and strain'

313 (Section 1), but for which the Gold Standard was 'characteristics of the organism' (Section 1).

314 For these terms, where the document was shown to lack in clarity, we adjusted the MITAP 
315 document accordingly; for example, we now state explicitly under 'characteristics of the

316 organism' that authors should indicate the source of purchase.

\section{Prevalence of MITAP data in extant papers}

319 The purpose of the MITAP document is to ensure that authors provide sufficient basic information about their tolAPC production protocol. An implicit assumption is that currently some or all of this information is not being routinely described. To test this assumption, we reviewed a number of papers about tolAPC, and for each, we determined whether the data required by the MITAP document was present or not.

In detail, forty-two tolAPC papers were selected (predominantly from members of AFACTT or from researchers well-known in the field), randomized and the first twenty were read in detail. For each section of MITAP, we determined whether the information required was either: directly stated in the paper or reference (table 1: green dots), whether it was possible to infer from other information given in the paper (table 1: yellow dots), whether all details were present or only partly (table 1: yellow dots) or whether the information was not present at all (table 1: red dots). For example, Section 1-ai of MITAP describes the species of the experimental organism. A paper with the phrase "human" or "Homo sapiens" would fall into the first category (included in the publication) one referring to "patients" would fall into the second category (included but some details missing). Many papers do not describe their experimental methodology, but instead refer to another paper ("as described previously"); in this case, we checked the paper up to two references away and if found, the information was considered as 'present' (table 1: green dots), if not it was considered as 'not present' (table 1: red dot). This work was performed by one of us (RS) who is a post-doctoral scientist with 6 years experience in the discipline. 


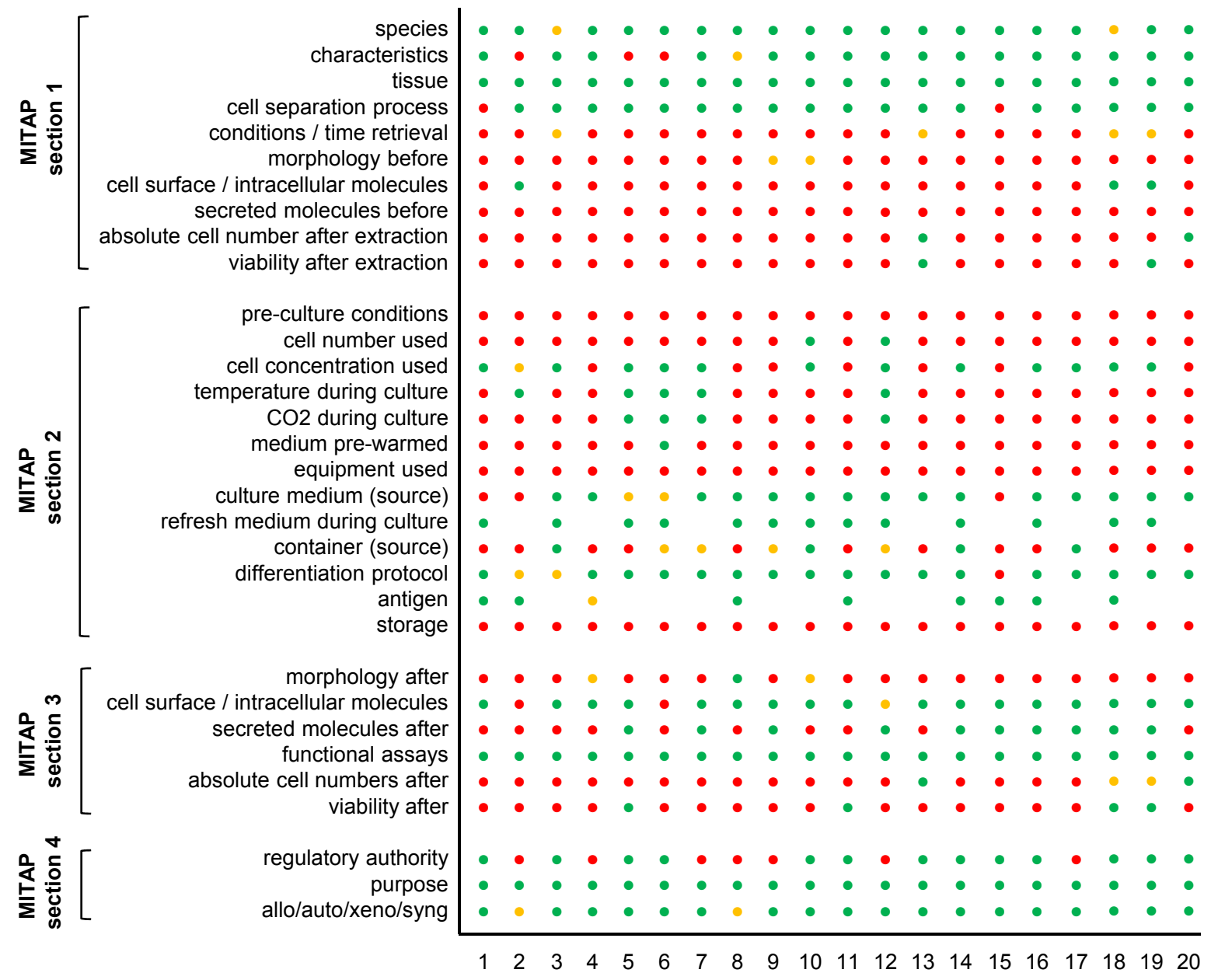

- category included in the publication

346 category included in the publication, but some details missing

347 category not included in the publication

348 -gap- information not present because not relevant for the publication

350 Results are shown in Table 1 . This shows that no papers have all the information required by

351 MITAP, and that most have substantial gaps. We can also see that while there is a relatively high

352 degree of conformity between different papers: e.g. species and growth media are commonly

353 reported; cell number, viability and culture container are rarely documented.

\section{Sustainability}


355 One significant issue with resources such as MITAP is their sustainability. Previous research has

356 shown that uniform resource identifiers (URIs; i.e. URLs or Web addresses) given in papers have

357 short half-lives (around a 25\% loss 3 years after publication ${ }^{15}$ ), and either move or become

358 inaccessible, in many cases before the papers have been published. This can happen either

359 because the web hosting is not stable, or fails, or because the domain providing the URI is not

360 maintained. To alleviate these difficulties with MITAP, neither the URIs nor the hosting of the

361 documents require active maintenance by the authors of this paper. We have used a permanent

362 identifier (http://w3id.org/ontolink/mitap) thereby providing a re-direction step. The primary

363 hosting of the resource is provided by Internet Archive (http://archive.org). Both of these

364 organisations have sustainability as their key aim. Maintenance of MITAP documents as live,

365 web-resources therefore requires no active contribution from the authors of this paper. Either

366 archive.org or w3id.org need to fail before MITAP cannot be maintained at the stated location.

368 We have also given explicit consideration to data formats for our documents. Resources are 369 available as Word docs and PDFs, providing typographical elegance and familiarity to the 370 community, but also an extremely simple HTML representation, ensuring vendor-neutrality and 371 future-proofing of the knowledge contained within them.

\section{Discussion}

374 The tolAPC community has advanced significantly in the last 15 years, from the initial

375 discoveries of how to culture APC with potent immune-regulatory properties, through to clinical

376 trials. At the current time, however, most investigators are operating as a "cottage industry":

377 individual labs working with different cell isolation techniques, procedures to induce stable

378 tolerogenic properties in the cells, and investigating different therapeutic outcomes. Against this

379 heterogeneous background, it becomes difficult to compare investigations between different

380 laboratories, raising concerns about reproducibility ${ }^{16,17}$.

382 The MITAP document that we have described here will, of course, not address all of these 383 issues, however as a minimum guidelines tool for reporting of protocols, it should at least 384 address the issue of data heterogeneity; different laboratories may still use different protocols to 
385

generate tolAPC, but at least we will be clear about this diversity, and have a greater understanding of what these differences are.

We have been careful in the process of building MITAP not to produce a document that is overly complex. Unlike many other minimum information documents, we have actively tested it with independent, essentially untrained annotators (test subjects). We have shown that it is possible, under these circumstances, to provide the information required in a clear and consistent manner. We believe that it should be possible for authors to complete the MITAP document in approximately 30 minutes. We also believe that this process should not be a burden to researchers; in our experience, we have adopted use of the MITAP document in writing our own papers, and believe that having a clear checklist to follow simplifies rather than confuses.

The process of building the MITAP document has been a highly collaborative one which has been a useful exercise in and of its own right: the degree of heterogeneity in the community has come as a surprise to many of those involved in this work. By analyzing extant papers, we have also shown that there is a discrepancy between the information that the community considers important and wants to receive about a TolAPC production protocol and the information that they actually report. For example, very few papers describe the viability of TolAPC at the end of the production process, although we consider it extremely likely that most researchers do actually measure this. Moreover, we can see no a priori reason why culture media largely is reported while culture container largely is not.

We believe that wide adoption could also contribute toward greater consideration of data management in the tolerogenic cell therapies community in general. For example, the MIATA project was recently extended to include NK cells (called MIANKA), and a similar approach could be taken by MITAP to include other regulatory cells, such as myeloid-derived suppressor cells, mesenchymal stem cells, and even T regulatory cells. Furthermore, unlike many minimum information efforts, we have considered upfront long-term sustainability of the documents and the URIs used to refer to them. Our sustainability plan is robust and does not require active involvement of any of the authors. 
416 Of course, there is much work remaining. The production of tolAPC is only a small part of the

417 overall process. Moreover, we are only attempting to standardize the reporting of protocols, and

418 not the standardisation of the protocols themselves. Finally, we currently have no common

419 location or database which can store the experimental data, such as exists for instance with

420 microarray or genomic data. MITAP is a first step, laying the groundwork for further

421 collaboration.

422

423 Funding statement

424 This work was supported by a grant from the European Cooperation in Science and Technology

425 (COST) for the AFACTT project (Action to Focus and Accelerate Cell-based Tolerance-

426 inducing Therapies; BM1305). COST is part of the EU Framework Programme Horizon 2020.

427

\section{Acknowledgement}

429 We would like to thank the volunteers (test subjects) who participated in testing MITAP. We

430 also thank Prof. Ranjeny Thomas for providing feedback on the manuscript and MITAP

431 document.

432

\section{References}

434 1. Amodio, G. \& Gregori, S. Human tolerogenic DC-10: perspectives for clinical applications. Transplant Res 1, 14 (2012).

2. Hutchinson, J.A., Riquelme, P. \& Geissler, E.K. Human regulatory macrophages as a cell-based medicinal product. Curr Opin Organ Transplant 17, 48-54 (2012).

3. Hilkens, C.M. \& Isaacs, J.D. Tolerogenic dendritic cell therapy for rheumatoid arthritis: where are we now? Clin Exp Immunol 172, 148-157 (2013).

4. Van Brussel, I. et al. Tolerogenic dendritic cell vaccines to treat autoimmune diseases: can the unattainable dream turn into reality? Autoimmun Rev 13, 138-150 (2014).

5. Creusot, R.J., Giannoukakis, N., Trucco, M., Clare-Salzler, M.J. \& Fathman, C.G. It's time to bring dendritic cell therapy to type 1 diabetes. Diabetes 63, 20-30 (2014).

6. Thomas, R. Dendritic cells as targets or therapeutics in rheumatic autoimmune disease. Curr Opin Rheumatol 26, 211-218 (2014). 
452 7. Morelli, A.E. \& Thomson, A.W. Orchestration of transplantation tolerance by regulatory

453

454

455

456

457

458

459

460

461

462

463

464

465

466

467

468

469

470

471

472

473

474

475

476

477

478

479

480

481

482

483

484

485

486

487 dendritic cell therapy or in-situ targeting of dendritic cells. Curr Opin Organ Transplant 19, 348-356 (2014).

8. Ten Brinke, A. et al. Clinical Use of Tolerogenic Dendritic Cells-Harmonization Approach in European Collaborative Effort. Mediators Inflamm 2015, 471719 (2015).

9. Giannoukakis, N., Phillips, B., Finegold, D., Harnaha, J. \& Trucco, M. Phase I (safety) study of autologous tolerogenic dendritic cells in type 1 diabetic patients. Diabetes Care 34, 2026-2032 (2011).

10. Benham, H. et al. Citrullinated peptide dendritic cell immunotherapy in HLA risk genotype-positive rheumatoid arthritis patients. Sci Transl Med 7, $290 \mathrm{ra} 287$ (2015).

11. Jauregui-Amezaga, A. et al. Intraperitoneal Administration of Autologous Tolerogenic Dendritic Cells for Refractory Crohn's Disease: A Phase I Study. J Crohns Colitis 9, 1071-1078 (2015).

12. Knudsen, T.B., Daston, G.P. \& Teratology, S. MIAME guidelines. Reprod Toxicol 19, 263 (2005).

13. Janetzki, S. et al. "MIATA"-minimal information about T cell assays. Immunity 31, 527528 (2009).

14. Britten, C.M. et al. Minimal information about T cell assays: the process of reaching the community of $\mathrm{T}$ cell immunologists in cancer and beyond. Cancer Immunol Immunother 60, 15-22 (2011).

15. Wren, J.D. URL decay in MEDLINE--a 4-year follow-up study. Bioinformatics 24, 1381-1385 (2008).

16. Ioannidis, J.P. Why most published research findings are false. PLoS Med 2, e124 (2005).

17. Boulton, G. Reproducibility: International accord on open data. Nature 530, 281 (2016). 